\title{
Control of signal coherence in parametric frequency mixing with incoherent pumps: narrowband mid-infrared light generation by downconversion of broadband amplified spontaneous emission source at $1550 \mathrm{~nm}$
}

\author{
Stefan Wabnitz, ${ }^{1,2, *}$ Antonio Picozzi, ${ }^{2}$ Alessandro Tonello, ${ }^{3}$ Daniele Modotto, ${ }^{1}$ and Guy Millot $^{2}$ \\ ${ }^{1}$ Department of Information Engineering, Università di Brescia, Brescia 25123, Italy \\ ${ }^{2}$ Laboratoire Interdisciplinaire Carnot de Bourgogne (ICB), Université de Bourgogne, Dijon 21078, France \\ ${ }^{3}$ Xlim, Université de Limoges, Limoges 87060, France \\ *Corresponding author: stefano.wabnitz@ing.unibs.it
}

Received August 15, 2012; accepted September 14, 2012;

posted September 24, 2012 (Doc. ID 174283); published October 24, 2012

\begin{abstract}
We study, with numerical simulations using the generalized nonlinear envelope equation, the processes of optical parametric and difference- and sum-frequency generation (SFG) with incoherent pumps in optical media with both quadratic and third-order nonlinearity, such as periodically poled lithium niobate. With ultrabroadband amplified spontaneous emission pumps or continua (spectral widths $>10 \mathrm{THz}$ ), group-velocity matching of a near-IR pump and a short-wavelength mid-IR (MIR) idler in optical parametric generation may lead to more than 15-fold relative spectral narrowing of the generated MIR signal. Moreover, the SFG process may also lead to 6-fold signal coherence improvements. When using relatively narrowband filtered noise pumps (e.g., spectral widths $<1 \mathrm{THz}$ ), the signal from optical parametric, sum-frequency, and difference-frequency generation has nearly the same spectral width as that of the incoherent pump. (c) 2012 Optical Society of America
\end{abstract}

OCIS codes: $190.4410,030.1640,190.4223$.

\section{INTRODUCTION}

Optical frequency conversion in quadratic nonlinear media using incoherent pump lasers has been studied for quite some time [1-ㅛ] ; in contrast to simple intuition, such resonant phase-sensitive processes may even lead to the generation of coherent waves [5-18]. The generation of a coherent signal from incoherent pumps may also occur through four-wave mixing in optical fibers [19]. In a quadratic medium, considering the purely one dimensional temporal problem and the limit case of small signals (i.e., no pump depletion), it has been predicted that the generation of a coherent signal may result in optical parametric generation (OPG) involving the downconversion of an incoherent pump [12-14]. The condition for obtaining such a coherent signal, besides the usual phase matching condition, is that the idler and pump group velocities should be locked; in this case it turns out that the signal growth is nearly independent of pump phase fluctuationsthe so-called phase-locking mechanism [12-14]. Therefore, the group-velocity mismatch (GVM) of the pump and idler waves was predicted to be a key parameter that permits control of the degree of coherence of the signal beam through the phase-locking of pump and idler, i.e., the pump incoherence appears to be effectively absorbed by the generated idler wave. Moreover, it was also predicted that the degree of signal coherence may even increase whenever the group-velocity dispersion (GVD) coefficients of the pump and idler are also matched $[14,15]$. It is also interesting to note that the mechanisms underlying these remarkable properties of the resonant interaction among three incoherent waves cannot be captured from the traditional formalism describing random nonlinear waves, i.e., the machinery of wave turbulence theory [20-22].

The possibility of controlling and improving the coherence of a signal in the process of parametric frequency conversion using incoherent pumps is presently of special applicative interest, in view of the availability of relatively inexpensive broadband noise sources based on filtered amplified spontaneous emission (ASE) in optical fiber amplifiers or continua generated by diode-pumped photonic crystal fibers [23].

Therefore, it is important and timely to analyze the validity of earlier predictions of GVM-based control of signal coherence in the regime of pump depletion and wideband frequency conversion by means of numerical simulations using the generalized nonlinear envelope equation (GNEE), which permits us to directly include the full frequency-dependent dispersion curve of the nonlinear crystal, as well as the influence of all competing parametric processes at once, thanks to a computational accuracy that goes beyond the usual slowly varying envelope approximation. Moreover, it is interesting to find out if the generation of a coherent signal is also possible whenever two independent incoherent pumps are involved, such as occurs in the processes of difference-frequency generation (DFG) and sum-frequency generation (SFG), respectively. 
In this work we analyze the coherence transfer properties between one or two incoherent pumps and the parametrically generated signal in optical media exhibiting both quadratic and third-order nonlinearity, such as periodically poled lithium niobate (PPLN). First of all, we confirm and substantially extend the predictions of [12-14], by showing that even ultrabroadband ASE noise pumps or continua (with spectral widths of several terahertz) that are exhibiting both intensity and phase fast temporal fluctuations may spontaneously downconvert into a highly coherent signal. For example, we numerically demonstrate that group-velocity (GV) matching between an intense near-IR (NIR) filtered ASE noise pump and short-wavelength mid-IR (MIR) idler quantum noise may lead to more than 15 -fold relative spectral narrowing of the OPG-generated long-wavelength MIR signal. Next we show that with two wideband ASE noise pumps, the SFG process may also lead to the narrowing of the signal spectral width. On the other hand, when using relatively narrowband ASE noise pumps (e.g., with a pump spectral width of $600 \mathrm{GHz}$ ), the signal from either SFG or DFG exhibits nearly the same spectral width as the pumps. In Section 2 we briefly summarize the GNEE approach to describe ultrawideband pulse propagation in quadratic and cubic nonlinear media. Subsequently, in Section 3 we present numerical simulation results of the basic three-wave frequency conversion processes in a PPLN waveguide, namely OPG, DFG, and SFG in the presence of incoherent pumps. In the case of OPG, we compare our numerical simulations with exact analytical predictions for the bandwidth of the generated signal, and we show that relatively good agreement is obtained. Finally, in Section $\underline{4}$ we present our conclusions.

\section{FULL FIELD NUMERICAL MODEL}

The propagation of a linearly polarized ultrabroadband light field in a quadratic and cubic nonlinear medium (i.e., the nonlinear polarization is $\left.P_{\mathrm{NL}}=P_{\mathrm{NL}}^{(2)}+P_{\mathrm{NL}}^{(3)}=\varepsilon_{0}\left(\chi^{(2)} E^{2}+\chi^{(3)} E^{3}\right)\right)$ with both quadratic and cubic nonlinearity may be usefully described in terms of a single GNEE for the complex $A(z, t)$, namely [24-26],

$$
\left[\partial_{z}-D+\frac{\alpha}{2}\right] A(z, t)=N p_{\mathrm{NL}}(z, t, A),
$$

where $D$ is the linear dispersion operator, $N \cong i \rho_{0}\left(1+i \tau_{\mathrm{sh}} \partial / \partial t\right)$, with $\rho_{0} \equiv \omega_{0} / 2 n_{0} c \varepsilon_{0}, n_{0}=n\left(\omega_{0}\right)$, and $\tau_{\text {sh }} \equiv 1 / \omega_{0}-\{\partial[\ln (n(\omega))] / \partial \omega\}_{\omega=\omega_{0}}, \omega_{0}$ is an arbitrary reference angular frequency, $n$ is the linear refractive index, $\alpha$ is the linear loss coefficient, and $\varepsilon_{0}$ is the vacuum permittivity. Moreover, the nonlinear polarization $p_{\mathrm{NL}}=p_{\mathrm{NL}}^{(2)}+p_{\mathrm{NL}}^{(3)}$, with

$$
\begin{aligned}
& p_{\mathrm{NL}}^{(2)}(z, t)=\varepsilon_{0} \chi^{(2)}\left(2|A|_{+}^{2} \exp \left(i \omega_{0} t\right)+A^{2} \exp \left(-i \omega_{0} t\right)\right) / 2, \\
& p_{\mathrm{NL}}^{(3)}(z, t)=\varepsilon_{0} \chi^{(3)}\left(3|A|^{2} A+A^{3} \exp \left(-2 i \omega_{0} t\right)\right) / 4
\end{aligned}
$$

where $|A|_{+}^{2}$ only contains frequency components with $\omega \geq 0$. The electric field is obtained from its envelope as $E(z, t) \equiv\left(A(z, t) \exp \left(-i \omega_{0} t\right)+c . c.\right) / 2, \quad$ and $\quad P_{\mathrm{NL}}(z, t) \equiv$ $\left(p_{\mathrm{NL}}(z, t) \exp \left(-i \omega_{0} t\right)+\right.$ c.c. $) / 2$.

Equation (1) may be easily numerically solved in the frequency domain as a set of coupled ordinary differential equations for the different frequency components of the field
$A(z, t)$. The second- and third-order nonlinearities are measured in terms of $d_{\text {eff }}=\chi^{(2)} / 2$ and the nonlinear refractive index $n_{2}=3 \chi^{(3)} / 8 n_{0}$, respectively.

\section{NUMERICAL RESULTS}

In this section, we present a series of numerical tests of the predictions of the linearized analysis of the three-wave model (see [12-14]) by directly solving the full GNEE [Eq. (1)] for describing both phase matched and possibly GV-matched frequency conversion processes in a PPLN waveguide.

Figure 1 shows the wavelength dependence of the group delay and group velocity dispersion $D$ for light propagating along the extraordinary axis in $\mathrm{LiNbO}_{3}$ [27]. As it can be seen in Fig. 1, the zero dispersion wavelength (ZDW) is found at about $2 \mu \mathrm{m}$; therefore, one may set the GVM between the pump and idler (or between the two pumps) to zero by symmetrically placing their wavelengths about the $\mathrm{ZDW}$ value, which also leads to almost equal group velocities for the two waves. In order to impose the proper phase matching among the three interacting wavelengths, we included in Eq. (1) a square-wave spatial modulation of the second-order nonlinear coefficient with different quasi-phase-matching (QPM) periods $\Lambda$; we used a quadratic nonlinear coefficient $d_{\text {eff }}=27 \mathrm{pm} / \mathrm{V}$ and the nonlinear refractive index $n_{2}=$ $5.3 \times 10^{-15} \mathrm{~cm}^{2} \mathrm{~W}^{-1}$.

For the incoherent pumps, we used the simplest possible model involving bandpass filtering [with a Gaussian filter transfer function $\left.T(f)=\exp \left(-\left(f-f_{p}\right)^{2} /\left(2 \sigma_{p}^{2}\right)\right)\right]$ of the amplitude of the white ASE noise field, by adding to each numerical frequency component a random variable with independent and Gaussian real and imaginary parts. Within the numerical window $T=21 \mathrm{ps}$, unless otherwise specified, the total energy of the incoherent pump was set equal to $4.6 \mathrm{~mJ} / \mathrm{cm}^{2}$, corresponding to an average intensity $\left\langle I_{p}\right\rangle=220 \mathrm{MW} / \mathrm{cm}^{2}$. For a $100 \mu \mathrm{m}^{2}$ effective area of the PPLN waveguide, this corresponds to an average power of $220 \mathrm{~W}$. Subsection 3.A presents a first summary of the results for the case of OPG, whereas in Subsection 3.B we study the case of DFG, and finally in Subsection 3.C we deal with SFG.

\section{A. Optical Parametric Generation}

Let us consider the OPG process, first of all, in order to test the validity of the predictions of [12-14] by means of the GNEE approach, and next, to explore the possibility of generating

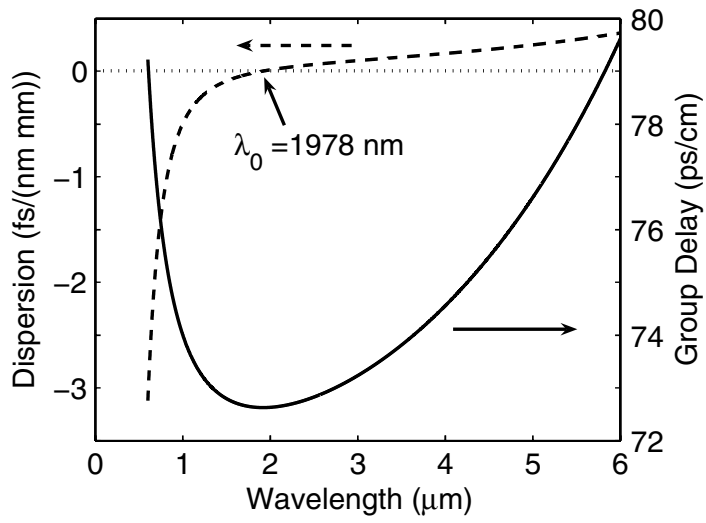

Fig. 1. Dependence of dispersion and group delay versus wavelength for propagation along the extraordinary axis of $\mathrm{LiNbO}_{3}$. 


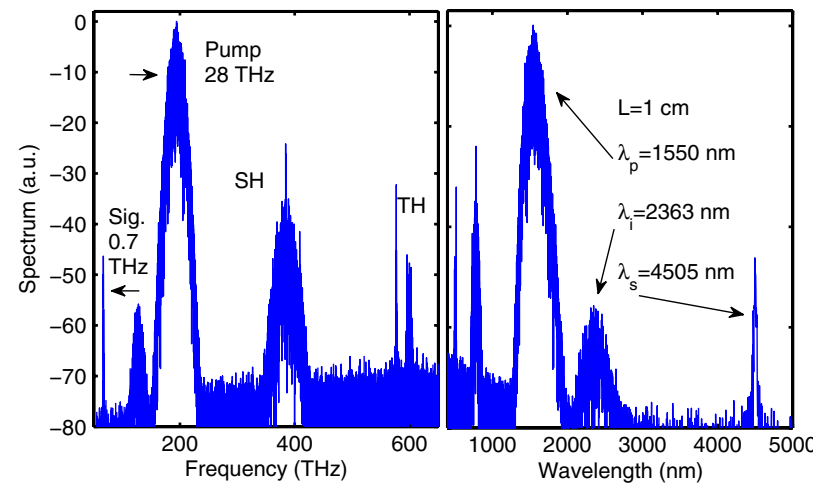

Fig. 2. (Color online) $28 \mathrm{THz}$ spectrum showing GV-matched OPG in an $L=1 \mathrm{~cm}$ long PPLN waveguide from a single incoherent pump at $\lambda_{p}=1550 \mathrm{~nm}$ (pump bandwidth of $28 \mathrm{THz}$, measured at $-10 \mathrm{~dB}$ from the spectral peak); QPM period $\Lambda=30.68 \mu \mathrm{m}$. Left (right): output spectrum versus frequency (wavelength).

a downconverted coherent signal by a GV-matched incoherent pump and idler in conditions well beyond the small-signal analysis of [12-14].

Figure 2 shows the quantum-noise-induced downconversion of a single wideband incoherent pump at $\lambda_{p}=$ $1550 \mathrm{~nm}$ with $\sigma_{p}=10 \mathrm{THz}$. In addition to the pump, the input field for Eq. (2) contained a one-photon-per-mode noise seed across the entire simulation bandwidth. The QPM period is $\Lambda=30.68 \mu \mathrm{m}$, so that the idler wavelength $\lambda_{i}=2363 \mathrm{~nm}$ leads to GV matching or velocity locking between the pump and the idler as discussed in [12-14]. Figure 2 shows that the spectral bandwidth (at $-10 \overline{\mathrm{dB}}$ from its peak) of the $\lambda_{s}=$ $4506 \mathrm{~nm}$ signal is equal to $0.7 \mathrm{THz}$, which is reduced by 40 times with respect to the corresponding $28 \mathrm{THz}$ (i.e., $224 \mathrm{~nm}$ ) pump bandwidth. On the other hand, Fig. 2 also shows the broadband content of the downconverted idler: its bandwidth is even larger than that of the pump. In fact, the idler fluctuations provide a sort of compensating phaseconjugate mirror image of the pump intensity and phase fluctuations, so that a coherent narrowband MIR signal results from the mixing of the broadband noise NIR pump and MIR idler [12-14].

In order to confirm the prediction that the incoherent pump and the noise-generated idler are indeed correlated by the wave mixing process, we numerically computed the pumpidler correlation function $\mu$, which describes the degree of their mutual coherence [28], namely,

$$
\mu(z)=\frac{\left|\Lambda_{0}(z)\right|}{\sqrt{\left\langle\left|a_{p}(z, t)\right|^{2}\right\rangle\left\langle\left|a_{i}(z, t)\right|^{2}\right\rangle}},
$$

where the mutual coherence function $\Lambda_{0}(z)=$ $\left\langle a_{p}(z, t) a_{s}^{*}(z, t)\right\rangle=\int\left\langle A_{p}(z, \omega) A_{s}^{*}(z, \omega)\right\rangle \mathrm{d} \omega, \quad$ and $\quad A_{p, s}(z, \omega)=$ $\int a_{p, s}(z, t) \exp (-i \omega t) \mathrm{d} t$. In order to extract the pump and idler fields, we have applied square-shaped frequency filters to the single-field GNEE solution.

In spite of the fact that the spectral amplitudes of the extracted pump and idler at $L=1 \mathrm{~cm}$ show no apparent mutual correlation (see the insets of Fig. 3), the dots joined by a blue solid curve in Fig. 3 clearly show that in the case of Fig. 2 there is a monotonous increase with the distance of the normalized correlation $\mu$ between the incoherent pump and the

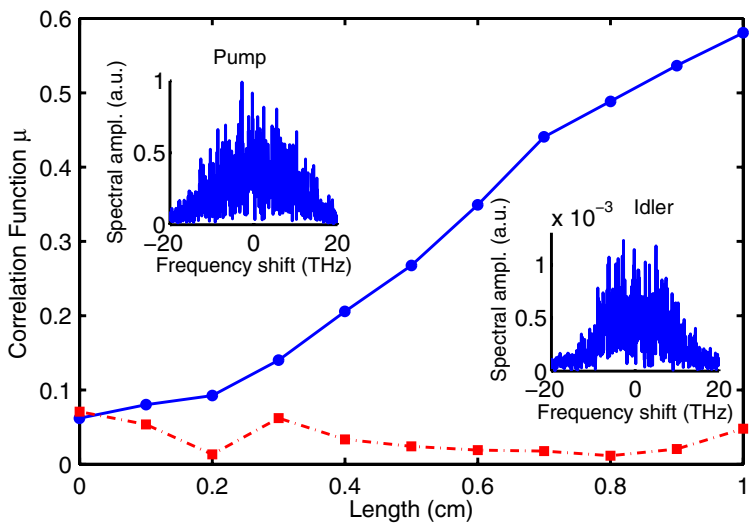

Fig. 3. (Color online) Growth of the correlation function $\mu$ between pump and idler versus propagation length. Dots joined by a blue solid curve (squares joined by a red dot-dashed curve): GV-matched (GVmismatched) case. Insets show the spectral amplitude of the pump and idler at $1 \mathrm{~cm}$ as in Fig. 2 .

parametrically generated idler up to the relatively high value of $\mu=0.6$ at $L=1 \mathrm{~cm}$ [29]. Notice in Fig. 3 that the evolution of $\mu$ exhibits a slight saturation with the propagation distance; indeed, the mutual correlation increases even further beyond $L=1 \mathrm{~cm}$.

Let us briefly compare the temporal fluctuations in the pump with the temporal dependence of the generated signal field. As shown in the left panel of Fig. 4, the temporal evolution of the input pump exhibits large and uncorrelated intensity fluctuations in addition to the simultaneous random phase fluctuations.

On the other hand, the right panel of Fig. 4 shows that the intensity fluctuations of the downconverted signal (as obtained by filtering the spectrum of Fig. 2 by a Gaussian bandpass filter centered at $\lambda_{s}=4506 \mathrm{~nm}$ with $\sigma_{p}=2 \mathrm{THz}$ ) are much smoother than the pump intensity fluctuations. Quite remarkably, a picosecond pulse of relatively high intensity with respect to the background fluctuations is formed in the MIR signal.

The generation of a coherent signal in the MIR spectral region from the downconversion of the incoherent NIR pump is preserved even in the strong conversion regime, where the pump is substantially depleted. Indeed, Fig. 5 shows the spectral amplitude of the generated field (in linear scale) at the output of an $L=4 \mathrm{~cm}$ long PPLN. As it can be seen, the peak spectral amplitude of the signal is nearly three times larger than the residual pump. Moreover, the spectral bandwidth
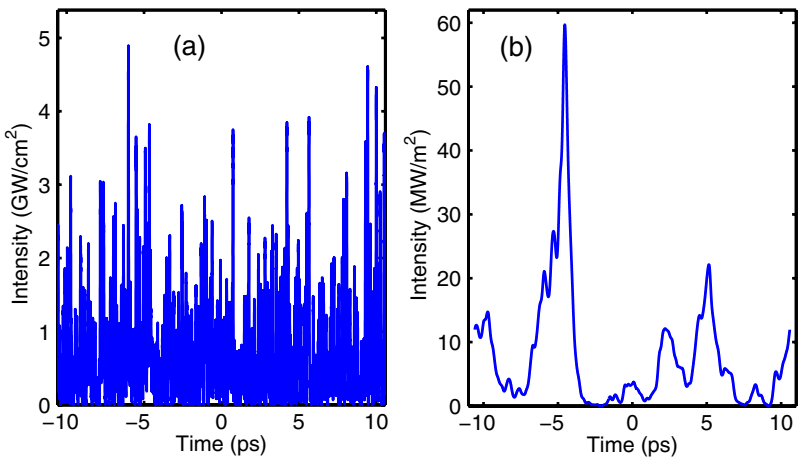

Fig. 4. (Color online) Time domain evolution of (a) input pump ASE noise intensity and (b) filtered output signal intensity at $4506 \mathrm{~nm}$. 


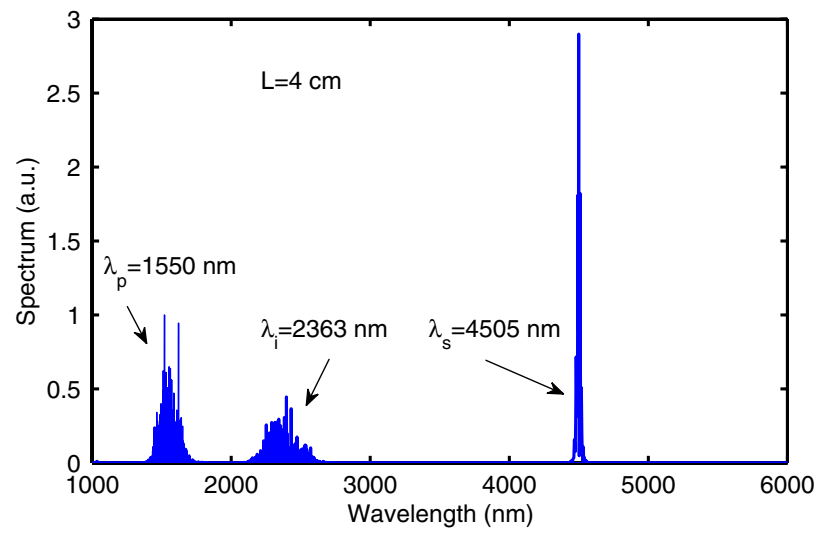

Fig. 5. (Color online) Same as in Fig. 2, for a PPLN length $L=4 \mathrm{~cm}$, but in a linear scale.

of the signal is unchanged with respect to the small-signal case of Fig. 2 .

Let us underline that the signal coherence recovery revealed by our simulations has been obtained in the presence of a broadband ASE noise pump, exhibiting rapid and virtually uncorrelated intensity and phase temporal fluctuations. Note that the NIR pump bandwidth of $28 \mathrm{THz}$ at $\lambda_{p}=1550 \mathrm{~nm}$ is equal to $15 \%$ of the carrier frequency, whereas the MIR signal bandwidth of $700 \mathrm{GHz}$ at $\lambda_{s}=4500 \mathrm{~nm}$ is equal to just $1 \%$ of the carrier frequency, so that a 15 -fold coherence improvement (relative spectral narrowing) is obtained through the GV-matched OPG process.

In order to verify the importance of the GV-matching condition leading to coherent MIR signal generation, we increased the QPM period $\Lambda=34.03 \mu \mathrm{m}$, so that the phasematched idler wavelength grows larger, up to $\lambda_{i}=2800 \mathrm{~nm}$, and the corresponding signal wavelength decreases to $\lambda_{s}=3472 \mathrm{~nm}$. In this case, the pump and idler are no longer GV-matched. As it can be seen from the left panel of Fig. 6, the signal bandwidth grows up to $4 \mathrm{THz}$, which is almost ten times larger than in the GV-matched case of Fig. 2. Indeed, the corresponding correlation function $\mu$ (squares joined by a dotdashed curve in Fig. 3) remains close to zero at all points in the crystal. Yet from the point of energy conversion efficiency from the pump to the idler, the ratio of total signal to pump energy at the crystal output remains nearly unchanged (at $10^{-6}$ ) at $L=1 \mathrm{~cm}$.

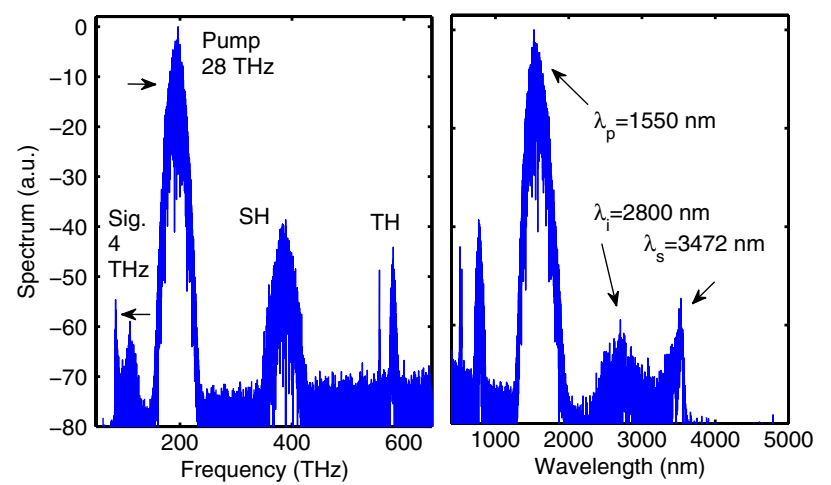

Fig. 6. (Color online) Same as in Fig. 2, for the GV-mismatched OPG in an $L=1 \mathrm{~cm}$ long PPLN waveguide from a single incoherent pump at $\lambda_{p}=1550 \mathrm{~nm}$ (pump bandwidth of $28 \mathrm{THz}$, measured at $-10 \mathrm{~dB}$ from the spectral peak); QPM period $\Lambda=34.03 \mu \mathrm{m}$.

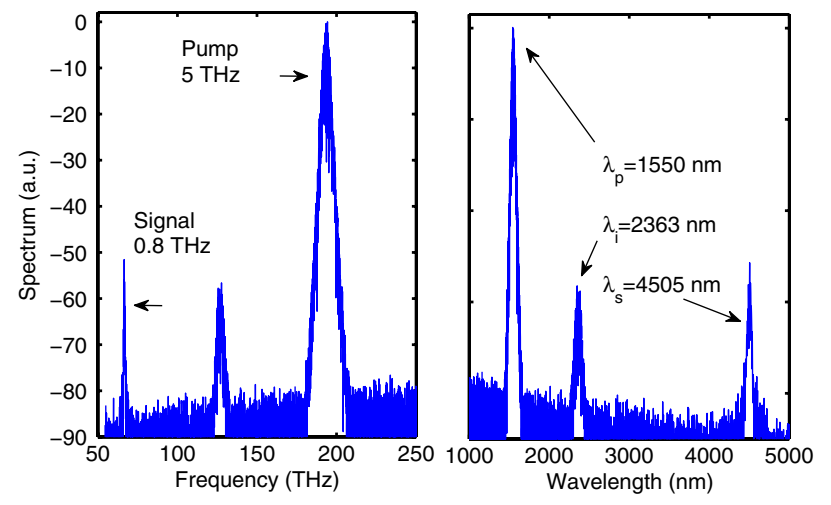

Fig. 7. (Color online) Same as in Fig. 2, but with a pump bandwidth of $5 \mathrm{THz}$, measured at $-10 \mathrm{~dB}$ from the spectral peak.

In Figs. $\underline{2}$ and $\underline{5}$ we demonstrated the generation of a coherent signal from an ultrabroadband (28 THz) ASE noise pump. Figure 7 shows that the signal bandwidth is virtually unchanged with respect to the case in Fig. 2 whenever the pump bandwidth is reduced down from 28 to $5 \mathrm{THz}$ (i.e., $\sigma_{p}=2 \mathrm{THz}$ ). However, as far as the energy conversion from the pump to the generated MIR idler and signal is concerned, narrowing the spectral width of the pump by 5 times (i.e., from the case of Figs. 2 to 7 ) leads to a 2.5 times efficiency improvement.

It is interesting to investigate in more detail the dependence of the output signal bandwidth on the bandwidth of the input ASE noise pump. For example, whenever a relatively narrowband incoherent pump (with a bandwidth of $0.6 \mathrm{THz}$, or $\sigma_{p}=200 \mathrm{GHz}$ ) is used, the spectral bandwidth of the signal remains virtually the same as that of the pump.

In order to provide a simple understanding of the spectral narrowing that is observed in the incoherently pumped parametric signal generation process of Fig. 2, it proves convenient to display the pump-frequency dependence of the dimensionless three-wave mixing parametric gain $G$ at high intensities [30], namely

$$
G=\Gamma^{2} L^{2} \sinh ^{2}(\gamma L) /(\gamma L)^{2},
$$

where $L$ is the waveguide length,

$$
\begin{aligned}
\gamma & =\left(\Gamma^{2}-\Delta k^{2} / 2\right)^{1 / 2}, \\
\Gamma^{2} & =\frac{\omega_{s} \omega_{i}}{c^{2} n_{s} n_{i}} I_{p} d^{2},
\end{aligned}
$$

$I_{p}$ is the pump intensity, $n_{s}$ and $n_{i}$ are the signal and idler refractive indexes at frequencies $\omega_{s}$ and $\omega_{i}$, respectively, $\Delta k=k_{p}-k_{s}-k_{i}-k_{\mathrm{QPM}}$ is the phase mismatch, and

$$
d=d_{\text {eff }} \sqrt{\frac{2 \eta_{0}}{n_{p}}}
$$

is the second-order nonlinear coefficient expressed in $\mathrm{m} / \mathrm{W}^{1 / 2}$. In Fig. 8 we compare, for a waveguide of length $L=$ $1 \mathrm{~cm}$ and pump intensity $I_{p}=2 \mathrm{GW} / \mathrm{cm}^{2}$, the variation of the gain $G$ with pump wavelength for the cases of GV-matched (for $\lambda_{p}=1550 \mathrm{~nm}$ ) pump and idler waves (see the top figure, where the QPM period is $\Lambda=30.68 \mu \mathrm{m}$ ) or GV-mismatched 


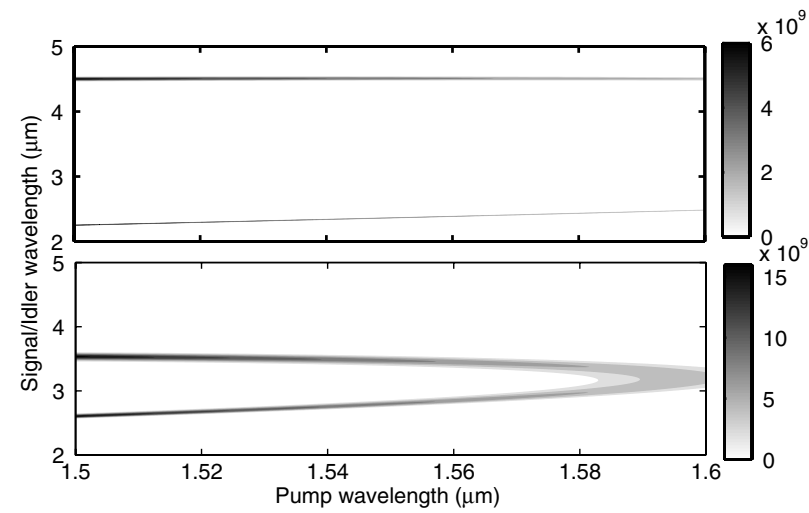

Fig. 8. Contour plot of the parametric gain $G$ for idler and signal wavelengths versus pump wavelength with (top) the QPM period $\Lambda=30.68 \mu \mathrm{m}$ or (bottom) $\Lambda=34.03 \mu \mathrm{m}$.

waves (see the bottom figure, where the QPM period $\Lambda=34.03 \mu \mathrm{m})$.

As can be seen from the top plot of Fig. $\underline{8}$, in the GVmatched case, the gain band of the signal remains relatively narrowband and it is virtually unchanged at $\lambda_{s}=4500 \mathrm{~nm}$ as the pump wavelength is varied by $100 \mathrm{~nm}$ across $1550 \mathrm{~nm}$. Indeed, whenever $\Delta k=0$ at a given pump frequency (say, $\left.\omega_{p}=\omega_{p 0}\right)$ such that the pump group-velocity $v_{p}$ equals the idler velocity $v_{i}$, then considering an equal shift frequency $\delta \omega$ of the pump and idler and supposing $k_{p} \cong k_{p 0}+\delta \omega / v_{p}$ and $k_{i} \cong k_{i 0}+\delta \omega / v_{i}$, one obtains that at the first order in $\delta \omega$ the mismatch $\Delta k$ remains equal to zero whenever the signal frequency is kept unchanged.

This qualitative understanding can be completed by a more rigorous analytical treatment of the parametric generation process that takes into account the incoherent nature of the pump wave. For this purpose, it proves convenient to resort to a more tractable model based on three-wave interaction, in which the evolution of the pump, signal, and idler components are ruled by three resonantly coupled equations. Moreover, a simple analysis based on the evaluation of the characteristic lengths reveals that GVD plays a negligible role in the three-wave mixing process as compared to the roles of the nonlinearity and the GV difference among the three waves. Then, neglecting chromatic dispersion, one can explicitly calculate the gain curve, $g(\omega)$, of the parametric instability for the signal wave in the presence of the incoherent pump [14 $\underline{15}]$ :

$$
g(\omega)=\operatorname{Re}\left\{-\Delta\left|\rho_{i}\right|+\sqrt{4-\left(\left(\rho_{s}-\rho_{i}\right) \omega \tau_{0}+i \Delta\left|\rho_{i}\right|\right)^{2}}\right\},
$$

where the variables $\rho_{s, i}=\left(v_{p}-v_{s, i}\right) / v_{s, i}$ denote the GV differences between the pump and the daughter (signal and idler) waves. In Eq. (7), the parameter $\Delta=\tau_{o} / \tau_{c}$ denotes the normalized spectral width of the pump wave, where $\tau_{o}=L_{\mathrm{N} L} / v_{p}, L_{\mathrm{NL}}=2 c n_{p} / \omega_{p} d \sqrt{\left\langle I_{p}\right\rangle}$ being the characteristic nonlinear length [31], and $\tau_{c}$ is the time correlation of the incoherent pump. We remark that the derivation of Eq. (7) implicitly assumes a Lorentzian-shaped pump spectrum, so that, strictly speaking, the comparisons of the theoretical predictions given by Eq. (7) with the numerical simulations discussed below are of qualitative nature.

Before discussing the numerical simulations reported above through Figs. $2-7$, let us briefly comment on the gain curve given in Eq. (7). First note that in the limit of a coherent pump, $\Delta \rightarrow 0$, Eq. (7) recovers the well-known parametric gain curve, where the factor $g(\omega=0)=2$ simply stems from the fact that the gain is defined as $g(\omega)=2 \operatorname{Re}[p(\omega)]$, where $p(\omega)$ is the growth rate of the parametric instability (see $[\underline{14}, \underline{15}]$ for details). More generally, the gain curve [Eq. (ㄱ)] describes different phenomena of parametric generation under incoherent excitation. We refer the reader to [14] for a discussion of different interesting limits of the gain $g(\omega)$ given in Eq. (7), e.g., the limit of the degenerate parametric configuration $\left(\rho_{s}=\rho_{i}\right)$ or the limit in which the velocities of the pump and idler waves are matched $\left(\rho_{i}=0\right)$, which leads to the phase-locking effect.

We reported in Fig. 9 the plots of the gain curve $g(\omega)$ and the corresponding signal spectrum $(\sim \exp [g(\omega) L])$ after propagation through an $L=1 \mathrm{~cm}$ crystal length with the numerical parameters of Figs. $\underline{2}$ and $\underline{6}$ discussed above. First of all, we remark in Fig. 9(a) that the incoherent nature of the pump affects the signal gain: one may notice a gain reduction by a factor 0.6 in the case of Fig. 6 (where the pump and idler waves are not GV-matched) as compared to the GV-matched case considered in Fig. 2. Note that a significant gain reduction is also clearly apparent in the numerical simulations of the GNEE. Besides this gain reduction, we also note in Fig. 9(a) the generation of a broad background spectral pedestal in the signal component.

On the other hand, the remarkable result is that the gain curve $g(\omega)$ plotted in Fig. $9(\mathrm{a})$ with the parameters of Fig. 2 is almost identical to the corresponding gain curve with a fully coherent pump, as one would expect from the phase-locking mechanism. Indeed, if the pump and idler group velocities are matched $\left(\rho_{i}=0\right)$, Eq. (7) reduces to $g(\omega)=\left[4-\left(\rho_{s} \omega \tau_{o}\right)^{2}\right]^{1 / 2}$, which does not depend on the pump incoherence parameter, $\Delta$. Actually, this expression of the gain coincides with the well-known expression obtained in the presence of a fully coherent pump wave. This confirms that, thanks to the GV matching among the pump and idler waves, the signal turns out to be efficiently amplified as if the pump were fully coherent. This velocity-locking effect merely explains why an increase of coherence in the pump does not lead to a coherence enhancement in the generated signal (compare Figs. 7
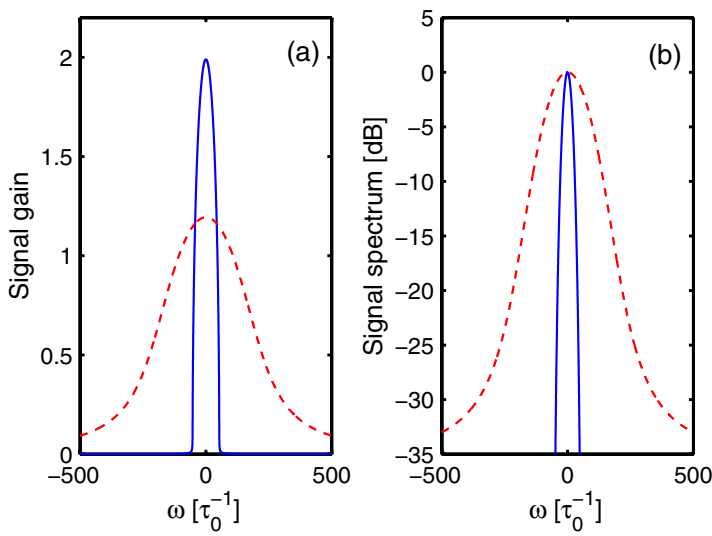

Fig. 9. (Color online) (a) Gain $g(\omega)$ of the signal wave in the presence of an incoherent pump obtained by plotting Eq. (7) with the parameters corresponding to Fig. $\underline{2}$ (solid blue curve) and Fig. $\underline{6}$ (dashed red curve), (b) corresponding signal spectra $(S(\omega) \sim$ $\exp [g(\omega) L])$, after propagation through an $L=1 \mathrm{~cm}$ crystal length [in $\log _{10}$ scale]. 
and 2 ), simply because in Figs. 2 and $\underline{7}$ the group velocities of the pump and idler waves are almost matched.

Finally, we note that there is a remarkable agreement between the analytical signal spectrum [see Fig. 9(b)] and the corresponding numerical simulations of the GNEE. Considering the group velocities $v_{j}$ given from the crystal dispersion curve (Fig. 1), a nonlinear length of $L_{\mathrm{NL}}=1.45 \mathrm{~mm}$, and a time correlation of $\tau_{c}=0.664 / \delta \nu$ [where $\delta \nu=2(\ln (2))^{1 / 2} \sigma_{p}$ is the FWHM of the pump spectrum], one obtains a bandwidth of the signal spectrum of $\sim 0.8 \mathrm{THz}$ for Fig. 2 and $\sim 4.3 \mathrm{THz}$ for Fig. 4 [at $-10 \mathrm{~dB}$, see Fig. 9(b)]. These spectral bandwidths are in good agreement with those obtained by integrating numerically the GNEE, i.e., $\sim 0.7$ and $4 \mathrm{THz}$, respectively. Note that the theoretical value of $0.8 \mathrm{THz}$ obtained with the parameters of Fig. 2 is also in agreement with the numerical simulations reported in Fig. 7, since, as discussed above, the coherence of the generated signal is not affected by pump incoherence for GV-matched pump and idler waves.

Note, however, that the coherence of the signal is slightly degraded in Fig. 7 as compared to Fig. 2, despite the fact that pump coherence is increased in Fig. 7 with respect to Fig. 2. This may be interpreted by remarking that while pump phase fluctuations are absorbed by the idler wave thanks to the phase-locking mechanism, the generation of the signal still remains affected by the intensity fluctuations of the pump. This point can be addressed through the analysis of the characteristic lengths associated to pump-signal GVM, $L_{\mathrm{GVM}}=$ $\tau_{c} /\left|\nu_{p}^{-1}-\nu_{s}^{-1}\right|$, and with the nonlinearity. In Fig. 7 one has $L_{\mathrm{GVM}} \sim 12 L_{\mathrm{NL}}$, whereas $L_{\mathrm{GVM}} \sim 0.2 L_{\mathrm{NL}}$ in Fig. 2 . This means that the intensity fluctuations of the pump are averaged out, thanks to the strong GVM among the pump and signal waves in Fig. 2, whereas in Fig. 7 the pump fluctuations are too slow to be averaged out and are thus partly transferred to the signal component. This effect of convection-induced averaging of intensity fluctuations has been discussed in detail in [13].

It is interesting to investigate next whether the signal coherence is robust in the presence of an input ASE noise idler in addition to the uniform white noise background. In this case DFG occurs between two ASE noise pumps, and its results will be described in Subsection 3.B.

\section{B. Difference-Frequency Generation}

Let us consider now the possibility of generating coherent (or spectrally narrowed) signals from the DFG process, that is, involving an input idler seed source in addition to the NIR ASE noise pump. In our simulations, we considered the DFG between two ASE noise pumps of generally different intensity. Figure 10 illustrates the output spectra that result in the same conditions as in the simulation of Fig. 2, where we have added at the input a filtered ASE noise idler with the same bandwidth as the NIR pump, but an energy $10^{-4}$ smaller than the pump. We underline that the (intensity and phase) fluctuations of the initial idler wave are generated independently from those of the pump wave.

As can be seen in Fig. 10, although the signal bandwidth remains virtually unchanged with respect to Fig. 2 , its spectral intensity grows larger by $25 \mathrm{~dB}$. The temporal profile of the filtered MIR signal (not shown here) reveals the presence of a main picosecond-duration peak similar to that of the right panel in Fig. 4, but with an intensity that is about $22 \mathrm{~dB}$ larger
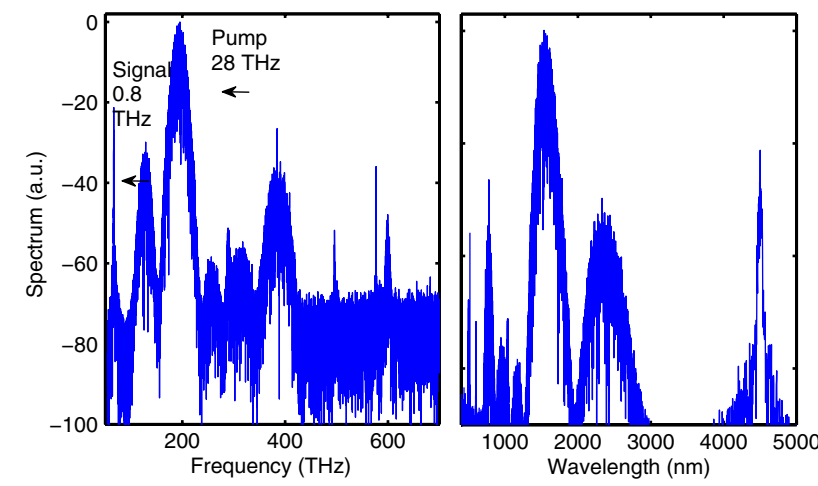

Fig. 10. (Color online) Same as in Fig. 2, but with an input idler ASE noise source of energy $10^{-4}$ smaller than the pump.

than that of Fig. 4. The result shows that the idler fluctuations, although independent of the pump fluctuations at the input, upon propagation evolve in such a way as to effectively match and cancel pump fluctuations in the generation of the signal idler.

This situation is shown even more dramatically in Fig. 11, which was obtained in the same conditions as in the simulation of Fig. 10, but with a broadband ASE noise idler energy $10^{-2}$ smaller than the pump. Figure 11 shows that again the signal bandwidth remains nearly unchanged with respect to Figs. 2 and 10, but its intensity grows larger by $20 \mathrm{~dB}$ with respect to Fig. 10. Indeed, the spectrum of Fig. 11 (see the linear scale shown in the inset) shows that the peak intensity of the signal grows $60 \%$ larger than the peak spectral intensity of the incoherent pump.

Let us finally consider the DFG of a coherent signal from two equal energy, GV-matched, relatively narrowband waves, a pump at $\lambda_{p}=1550 \mathrm{~nm}$, and an idler at $\lambda_{i}=2363 \mathrm{~nm}$, with the common bandwidth $\sigma_{p}=200 \mathrm{GHz}$. In this case (not shown here), one obtains a spectral width of $0.4 \mathrm{THz}$ of the DFG signal at $4500 \mathrm{~nm}$, which is nearly equal to the output spectral width of the long-wavelength pump and half the output spectral width of the short-wavelength pump. It is remarkable that the signal spectral bandwidth is nearly unchanged with respect to the cases shown in Figs. 10 and 11, where the input pump bandwidths were 50 times larger. Indeed, in the lower limit the spectral bandwidth of the signal is set by the intrinsic frequency width of the nonlinear parametric gain $G$ of Eq. (3).

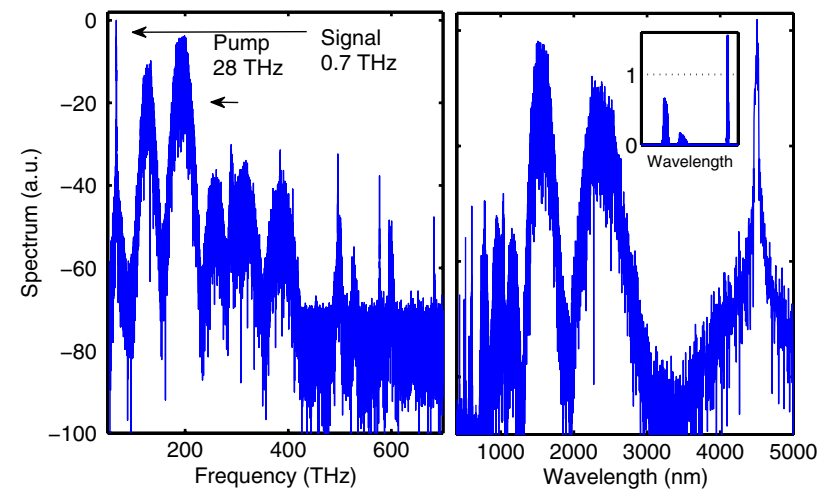

Fig. 11. (Color online) Same as in Fig. 2, but with an input idler ASE noise source of energy $10^{-2}$ smaller than the pump. Inset: output spectrum in linear scale. 


\section{Sum-Frequency Generation}

It is interesting to see if the SFG process may also lead to signal spectral narrowing whenever incoherent pumps are employed, as may be predicted by extending to this case the small-signal analysis of [12-14]. At first we simulate the SFG at $\lambda_{s}=936 \mathrm{~nm}$ with an equal intensity (with the average value of $220 \mathrm{MW} / \mathrm{cm}^{2}$ ) GV-matched incoherent pump at $\lambda_{p}=$ $1550 \mathrm{~nm}$ and idler at $\lambda_{i}=2363 \mathrm{~nm}$, respectively. The corresponding QPM period is $\Lambda=27.19 \mu \mathrm{m}$. Both pump and idler have the same spectral width parameter $\sigma_{p}=2 \mathrm{THz}$ : in this case the signal spectral width $(5 \mathrm{THz}$, as measured at $-10 \mathrm{~dB}$ from the spectral peak) turns out to be only slightly narrower than the spectral width of the pumps, which is equal to about $7 \mathrm{THz}$.

As we have done for the OPG process shown in Fig. 2 , let us now consider the SFG with an ultrabroadband pump at $\lambda_{p}=$ $1550 \mathrm{~nm}$ with $\sigma_{p}=10 \mathrm{THz}$ and an average intensity of $220 \mathrm{MW} / \mathrm{cm}^{2}$. Whenever no idler at $\lambda_{i}=2363 \mathrm{~nm}$ (except for the white quantum noise seed) is injected, the SFG process is effectively quenched by the growth of the second harmonic of the $1550 \mathrm{~nm}$ pump: no sum-frequency signal at $\lambda_{s}=936 \mathrm{~nm}$ is observed in the output spectra.

In order to restore the GV-matched SFG process, it is necessary to seed the idler: we did that by injecting, alongside the pump, a broadband ASE noise seed centered at the idler wavelength $\lambda_{i}=2363 \mathrm{~nm}$ with the same bandwidth as the pump ( $\sigma_{p}=10 \mathrm{THz}$, or a bandwidth of $28 \mathrm{THz}$, measured at $-10 \mathrm{~dB}$ from the spectral peak).

Indeed, Fig. 12 (where an idler ASE noise seed with a total energy 100 times less than the $1550 \mathrm{~nm}$ pump energy) shows that in this case the seeded SFG process leads to the significant growth of a $\lambda_{s}=936 \mathrm{~nm}$ signal. Quite remarkably, Fig. 12 shows that the coherence of the SFG signal is increased (or, equivalently, its spectral width is decreased) by almost a factor of four with respect to that of the pump. In fact, the signal spectral width of $7.5 \mathrm{THz}$ means a bandwidth of only $2.3 \%$ of its carrier frequency, which entails a coherence improvement in the signal in excess of six times with respect to the $1550 \mathrm{~nm}$ pump. Such coherence enhancement may be ascribed to the mutual compensation of the pump and idler phase and amplitude fluctuations, which appears to remain in place for the SFG process, although with a reduced efficiency with respect to the OPG or DFG case.

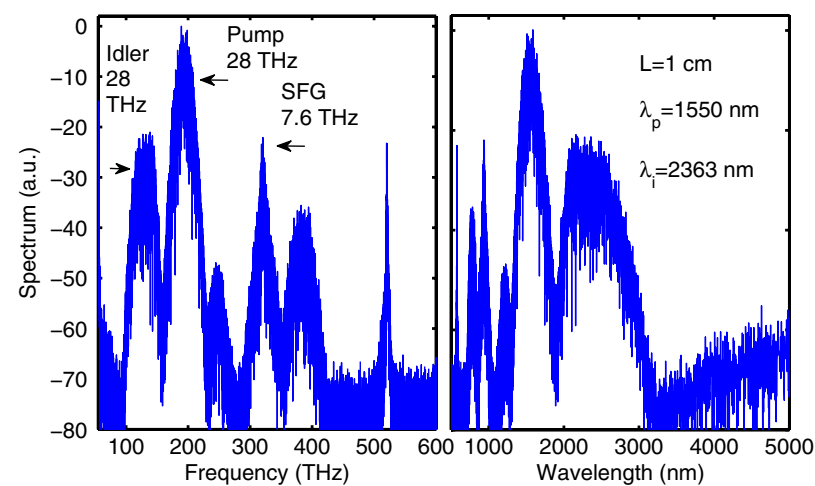

Fig. 12. (Color online) GV-matched SFG in a $1 \mathrm{~cm}$ long PPLN sample from an incoherent, $28 \mathrm{THz}$ wide ASE noise pump at $\lambda_{p}=1550 \mathrm{~nm}$ and an idler (of energy $10^{-2}$ less than the pump) at $\lambda_{i}=2363 \mathrm{~nm}$, respectively. The signal is generated at $\lambda_{s}=938 \mathrm{~nm}$ with a $7.5 \mathrm{THz}$ spectral width, measured at $-10 \mathrm{~dB}$ from the peak.
Figure 12 involves SFG with GV-matched pumps. On the other hand, in Fig. 13 we replaced the long-wavelength pump (idler) of Fig. 12 with a new, relatively short-wavelength pump (idler) at $127 \overline{\mathrm{nm}}$. Correspondingly, we set the QPM period $\Lambda=15 \mu \mathrm{m}$, so that the pumps are phase matched to a sumfrequency signal at $\lambda_{s}=700 \mathrm{~nm}$. In this case the GVM between $\lambda_{p}$ and $\lambda_{i}$ is nonzero; moreover both pumps have the same bandwidth, $\sigma_{p}=10 \mathrm{THz}$, but the energy of the shortwavelength pump at $\lambda_{i}=1276 \mathrm{~nm}$ is set to be 100 times less than the long-wavelength pump at $1550 \mathrm{~nm}$. As is shown in Fig. 13 , the spectral width of the generated signal is equal to $3.9 \mathrm{THz}$, which is almost halved with respect to the sumfrequency signal bandwidth in the GV-matched case of Fig. 12. It turns out that the sum-frequency signal bandwidth remains nearly unchanged when the spectral bandwidth of the idler and pump is reduced by five times with respect to Figs. 12 and $\underline{13}$, namely, if we set $\sigma_{p}=2 \mathrm{THz}$.

Until now we considered SFG with ultrabroadband incoherent pumps; when reducing the incoherent pump bandwidth further by decreasing the optical filter bandpass (e.g., by 10 times down to $\sigma_{p}=200 \mathrm{GHz}$ ), one finds that the output signal bandwidth at $700 \mathrm{~nm}$ with GV-matched (as in Fig. 12) or -mismatched pumps (as in Fig. 13) remains nearly equal to the output bandwidth of the pumps.

In analogy with our discussion in Subsection 3.A for the OPG case, consider the effect on the sum-frequency signal of the approximate conservation of the linear phase mismatch $\Delta k=0$ as both the pump and idler frequencies are varied. If $\Delta k=0$ at a given pump frequency (say, $\omega_{p}=\omega_{p 0}$ ) such that the pump group-velocity $v_{p}$ equals the idler velocity $v_{i}$, and opposite frequency shifts $+\delta \omega$ and $+\delta \omega$ are imposed to the pump and idler, respectively, by supposing $k_{p} \cong k_{p 0}+\delta \omega /$ $v_{p}$ and $k_{i} \cong k_{i 0}-\delta \omega / v_{i}$, one obtains that at the first order in $\delta \omega$, the mismatch $\Delta k$ remains equal to zero whenever the signal frequency is kept unchanged, which explains the generation of relatively narrowband sum-frequency signals. However, this simple reasoning does not explain our numerical observation of enhanced sum-frequency signal spectral narrowing in the GV-mismatched case as in Fig. 13. We therefore leave a more detailed analytical investigation of the SFG case to a forthcoming study.

In conclusion, our numerical study of SFG indicates that in contrast with the OPG and DFG case, the pump GVM does not

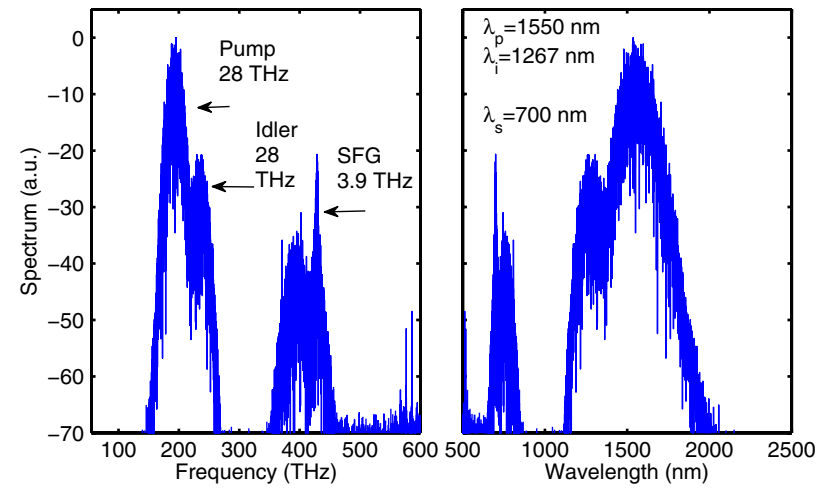

Fig. 13. (Color online) GV-mismatched SFG with an incoherent idler at $\lambda_{i}=1276 \mathrm{~nm}$ (of energy $10^{-2}$ less than the pump) and a pump at $\lambda_{p}=1550 \mathrm{~nm}$, respectively (with bandwidths of $28 \mathrm{THz}$ ); the QPM period is $\Lambda=15 \mu \mathrm{m}$. The $700 \mathrm{~nm}$ signal spectral width is equal to 3.9 THz. 
play a significant role in determining the spectral width of the sum-frequency signal. In any case, we observed that the signal bandwidth may be significantly reduced with respect to the pump bandwidth in the case of ultrabroadband incoherent pumps or continua, with bandwidths equal to or larger than $10 \mathrm{THz}$.

\section{CONCLUSIONS}

We studied the relative bandwidth of pumps and parametrically generated signals in PPLN with incoherent pumps of different spectral widths. We have shown that whenever ultrabroadband ASE noise pumps or continua are employed (e.g., for pump spectral widths above $10 \mathrm{THz}$ ), the mutual cancellation of velocity-locked pump and idler fluctuations leads to a signal coherence improvement (or spectral narrowing) in excess of 15 times for both the OPG and DFG processes, whereas the SFG exceeds coherence improvements of about 6 times. On the other hand, when using relatively narrowband filtered ASE noise pumps (with spectral widths of the order of $1 \mathrm{THz}$ or less), parametric signals emerging from the abovementioned processes exhibit nearly identical spectral widths of the pumps, nearly independently of the condition of GV matching between the pump and idler.

\section{ACKNOWLEDGMENTS}

We acknowledge stimulating discussions with V. Couderc and helpful remarks by T. Hansson. This work was carried out with support from the Fondazione Cariplo grant Nb. 20092730, the Conseil Régional de Bourgogne, and the iXCore Foundation.

\section{REFERENCES AND NOTES}

1. S. H. Harris, "Threshold of multimode parametric oscillators," IEEE J. Quantum Electron. 2, 701-702 (1966).

2. J. Ducuing and N. Bloembergen, "Statistical fluctuations in nonlinear optical processes," Phys. Rev. 133, A1493-A1502 (1964).

3. H. Hsu, "Parametric interactions involving multiple elementary scattering processes," J. Appl. Phys. 38, 1787-1789 (1967).

4. R. H. Byer, M. K. Oshman, J. F. Young, and S. E. Harris, "Visible CW parametric oscillator," Appl. Phys. Lett. 13, 109-111 (1968).

5. A. Piskarskas, V. Smilgevicius, and A. Stabinis, "Optical parametric oscillation excited by an incoherent conical beam," Opt. Commun. 143, 72-74 (1997).

6. A. Marcinkevičius, A. Piskarskas, V. Smilgevičius, and A. Stabinis, "Parametric superfluorescence excited in a nonlinear crystal by two uncorrelated pump beams," Opt. Commun. 158, 101-104 (1998).

7. A. Dubietis, R. Danielius, G. Tamošauskas, and A. Piskarskas, "Combining effect in a multiple-beam-pumped optical parametric amplifier," J. Opt. Soc. Am. B 15, 1135-1139 (1998).

8. A. Piskarskas, V. Smilgevicius, A. Stabinis, and V. Vaicaitis, "Spatially cumulative phenomena and output patterns in optical parametric oscillators and generators pumped by conical beams," J. Opt. Soc. Am. B 16, 1566-1578 (1999).

9. C. Montes, W. Grundkötter, H. Suche, and W. Sohler, "Coherent signal from incoherently cw-pumped singly resonant Ti:LiNbO3 integrated optical parametric oscillators," J. Opt. Soc. Am. B 24, 2796-2806 (2007)

10. G. Tamosauskas, A. Dubietis, G. Valiulis, and A. Piskarkas, "Optical parametric amplifier pumped by two mutually incoherent laser beams," Appl. Phys. B 91, 305-307 (2008).
11. A. Piskarskas, V. Pyragaite, and A. Stabinis, "Generation of coherent waves by frequency up-conversion and down-conversion of incoherent light," Phys. Rev. A 82, 053817 (2010).

12. A. Picozzi and M. Haelterman, "Parametric three-wave soliton generated from incoherent light," Phys. Rev. Lett. 86, 2010-2013 (2001).

13. A. Picozzi, C. Montes, and M. Haelterman, "Coherence properties of the parametric three-wave interaction driven from an incoherent pump," Phys. Rev. E 66, 056605 (2002).

14. A. Picozzi and P. Aschieri, "Influence of dispersion on the resonant interaction between three incoherent waves," Phys. Rev. E 72, 046606 (2005).

15. A. Picozzi and M. Haelterman, "Condensation in Hamiltonian parametric wave interaction," Phys. Rev. Lett. 92, 103901 (2004).

16. C. Montes, A. Picozzi, and K. Gallo, "Ultra-coherent signal output from an incoherent cw-pumped singly resonant optical parametric oscillator," Opt. Commun. 237, 437-449 (2004).

17. G. Strömqvist, V. Pasiskevicius, C. Canalias, and C. Montes, "Coherent phase-modulation transfer in counterpropagating parametric down-conversion,” Phys. Rev. A 84, 023825 (2011).

18. G. Strömqvist, V. Pasiskevicius, C. Canalias, P. Aschieri, A. Picozzi, and C. Montes, "Temporal coherence in mirrorless optical parametric oscillators," J. Opt. Soc. Am. B 29, 1194-1202 (2012).

19. Y. Yan and C. Yang, "Coherent light wave generated from incoherent pump light in nonlinear Kerr medium,” J. Opt. Soc. Am. B 26, 2059-2063 (2009).

20. V. E. Zakharov, V. S. L'vov, and G. Falkovich, Kolmogorov Spectra of Turbulence I (Springer, 1992).

21. See, e.g., A. Picozzi, "Toward a nonequilibrium thermodynamic description of incoherent nonlinear optics," Opt. Express 15, 9063-9083 (2007).

22. S. Lagrange, H. R. Jauslin, and A. Picozzi, "Thermalization of the dispersive three-wave interaction," Europhys. Lett. 79, 64001 (2007).

23. F. Baronio, M. Conforti, C. De Angelis, M. Andreana, A. Tonello, and V. Couderc, "Tunable light source from large band conversion of continuum in a quadratic crystal," Laser Phys. Lett. 9, 359-362 (2012).

24. M. Conforti, F. Baronio, and C. De Angelis, "Nonlinear envelope equation for broadband optical pulses in quadratic media," Phys. Rev. A 81, 053841 (2010).

25. M. Conforti, F. Baronio, and C. De Angelis, "Ultrabroadband optical phenomena in quadratic nonlinear media," IEEE Photon. J. 2, 600-610 (2010).

26. S. Wabnitz and V. V. Kozlov, "Harmonic and supercontinuum generation in quadratic and cubic nonlinear optical media," J. Opt. Soc. Am. B 27, 1707-1711 (2010).

27. Dispersion data taken from Handbook of Optics, M. Bass, ed., 2nd ed. (McGraw-Hill, 1994), Vol. 2.

28. See, e.g., L. Mandel and E. Wolf, Optical Coherence and Quantum Optics (Cambridge University, 1995).

29. Note that the nonzero value of $\mu$ at the crystal input $z=0$ originates in the relatively small temporal window considered in the simulations, $T=21 \mathrm{ps}$. By increasing $T$, one reduces the corresponding grid discretization in frequency space, $d \omega=2 \pi / T$, which thus reduces the value of $\mu(z=0)$. In other terms, in the thermodynamic limit (the limit in which the power $P$ and $T$ tend to infinity keeping constant $P / T$ ), the mutual coherence $\mu$ tends to zero. However, given the complexity of the GNEE, only a relatively small temporal window can be considered in the numerical integration.

30. P. S. Kuo, K. L. Vodopyanov, M. M. Fejer, D. M. Simanovskii, X. Yu, J. S. Harris, D. Bliss, and D. Weyburne, "Optical parametric generation of a mid-infrared continuum in orientation-patterned GaAs," Opt. Lett. 31, 71-73 (2006).

31. In our examples, the nonlinear length associated with the nonlinear Kerr effect is about 1000 times longer than $L_{\mathrm{nl}}$ and can be neglected in our analysis. 